\title{
Sub-nanometer thin oxide film sensing with localized surface phonon polaritons
}

Rodrigo Berte, ${ }^{\dagger, \ddagger} @$ Christopher R. Gubbin, ${ }^{\boldsymbol{\Phi}, @ ~ V i r g i n i a ~ D . ~ W h e e l e r, ~}{ }^{\S}$ Alexander J.

Giles, ${ }^{\S}$ Vincenzo Giannini, ${ }^{\dagger}, \|$ Stefan A. Maier, ${ }^{\dagger, \perp}$ Simone De Liberato, ${ }^{\circledR}$ and Joshua D. Caldwell*,\#,§

$\dagger$ The Blackett Laboratory, Department of Physics, Imperial College London, London SW7 2AZ, United Kingdom

$\ddagger$ CAPES Foundation, Ministry of Education of Brazil, Brasilia, DF 70040-020, Brazil

ISchool of Physics and Astronomy, University of Southampton, Southampton, SO17 1BJ, United Kingdom

$\S U . S$. Naval Research Laboratory, Washington, DC, USA.

|| Instituto de Estructura de la Materia (IEM-CSIC), Consejo Superior de Investigaciones Científicas, Serrano 121, 28006 Madrid, Spain

$\perp$ Nanoinstitut München, Fakultät für Physik, Ludwig-Maximilians-Universität München, 80799 München, Germany

\#Department of Mechanical Engineering, Vanderbilt University, Nashville, Tennessee 37205 United States

@ Contributed equally to this work

E-mail: josh.caldwell@vanderbilt.edu

\begin{abstract}
Chemical sensing methods based on surface polaritonic resonances stem from their intense near fields and resultant sensitivity to changes in local refractive index. Polar
\end{abstract}


dielectric crystals (e.g. $\mathrm{SiC}, \mathrm{hBN}$ ) support surface phonon polaritons (SPhPs) from the mid-infrared to terahertz range with mode volumes and quality factors exceeding the best case scenario attained by plasmonic counterparts, making them strong candidates for resonant surface-enhanced infrared spectroscopy (SEIRA). We report on the behaviour of SPhP resonances of SiC nanopillars following the incorporation of sub- and nanometric coatings of $\mathrm{Al}_{2} \mathrm{O}_{3}$ and $\mathrm{ZrO}_{2}$ obtained by atomic layer deposition. Concurrent anomalous red and blue-shifts of SPhP resonances were observed upon deposition of sub-nanometric $\mathrm{Al}_{2} \mathrm{O}_{3}$ films, with shift direction dictated by the mode position relative to the ordinary longitudinal optic (LO) phonon of $\mathrm{Al}_{2} \mathrm{O}_{3}$. These concurrent shifts, which are attributed to coupling to the Berreman mode of the $\mathrm{Al}_{2} \mathrm{O}_{3}$ layer, persist for thicker films and are correctly predicted by numerical calculations employing the measured $\mathrm{Al}_{2} \mathrm{O}_{3}$ permittivity. Deposition of $\mathrm{ZrO}_{2}$, whose phonon resonances are detuned from the $\mathrm{SPhPs}$, also led to anomalous blue-shifts of transverse and longitudinal SPhP resonances around $900 \mathrm{~cm}^{-1}$ for films up to $\approx 1.5 \mathrm{~nm}$, reversing to the canonical red-shift for thicker layers. These anomalous shifts were not reproduced numerically using the measured $\mathrm{ZrO}_{2}$ permittivity and provide evidence for a localized surface state, which when modelled as a simple Lorentz oscillator, provide semi-quantitative agreement with experimental results. In addition, predicted shifts for thicker layers may thus provide a tool for real-time monitoring of ultrathin film growth.

\section{Keywords}

Surface Phonon Polaritons, Oxides, Atomic layer Deposition, Chemical Sensing, $\mathrm{ZrO}_{2}, \mathrm{Al}_{2} \mathrm{O}_{3}$

In dielectric photonic systems, the interaction between nanoscopic quantum emitters and light is inherently weak as a result of the dimensional mismatch between diffraction limited photonic modes and strongly localized emitters. This problem is traditionally overcome by exploitation of high quality factor optical microcavities, allowing photons to transit an emitter many times before escaping the system. Such tuning of the light-matter interaction 
allows for enhanced spontaneous emission via the Purcell effect ${ }^{?}$ and, where the light-matter interaction rate exceeds the cavity linewidth, for a strong coupling regime with the formation of quasiparticles termed polaritons.?

An alternative way to achieve strong light-matter interactions is to circumvent the diffraction limit by transiently storing photonic energy in coherent oscillations of charges. In the case of an electronic charge, the resulting modes are termed plasmons. ${ }^{?}$ In this case energy exists on an intermediate, morphologically dependant length scale, potentially orders of magnitude below that of the free-space photon. Increased spatial overlap between photons and emitters and the resulting near-field enhancements have yielded drastic efficiency improvements of naturally weak phenomena such as Raman-scattering where single molecule sensing has been achieved. ' As the confinement is mediated by the electrons, tighter localisation is always accompanied by increased Ohmic losses, ${ }^{?} ?$ necessitating complex architectures to separate the field hotspot from the host metal ${ }^{?}$ or incorporate external gain media. ${ }^{?}$ An emergent alternative are surface phonon polaritons (SPhPs), modes formed by hybridisation of free photons with coherent lattice oscillations of a polar dielectric crystal. ${ }^{\text {? }}$ Supported between the longitudinal ( $\mathrm{LO}$ ) and transverse (TO) optic phonon frequencies in a narrow, material and crystalline structure-dependent, spectral region called the Reststrahlen band. Like plasmons, these modes have characteristic lifetimes determined by that of their matter component, however, as optic phonons usually possess lifetimes three orders of magnitude longer than collective electron resonances in noble metals, SPhPs offer benefits commensurate with plasmons (e.g. subdiffractional confinement of light), while exhibiting significantly lower losses.

The morphologically dependent resonances supported by polar dielectrics ? ? have been exploited for demonstration of extraordinary transmission, ${ }^{?}$ sub-diffraction imaging, ${ }^{\text {hy- }}$ perlensing, ${ }^{?} ?$ surface-enhanced infrared absorption ${ }^{?}$ and have been proposed as a platform for mid-infrared nonlinear optics. ? ? In recent years tailored localized SPhP resonances with exceptionally narrow linewidths were demonstrated in user-defined arrays of nanopillars on 
substrate. ? These narrow linewidths permit the strong coupling of localized and propagating SPhPs, thereby allowing for predictive dispersion tuning. ? In addition predictions of extreme near field enhancements, ${ }^{\text {? }}$ far exceeding the theoretical limit for plasmonic systems, would make such localized modes highly sensitive to very small changes in the local environment. Furthermore, the phonon-based nature of the SPhPs coupled with the strong surface sensitivity of the polaritonic near-fields infers that small changes to the chemical bonding at the surfaces of SPhP nanostructures could have large impact upon the resonant behavior. While prior works have demonstrated that changing the local environment of a polaritonic nanostructure, such as the $\mathrm{SiC}$ nanopillars discussed here, results in a spectral red-shift of the resonance due to the increase in the effective local index of refraction of the ambient, ${ }^{?}$ ? here we report on the observation of anomalous red and blue-shifts in the localized SPhP resonances using ultrathin alumina $\left(\mathrm{Al}_{2} \mathrm{O}_{3}\right)$ and zirconia $\left(\mathrm{ZrO}_{2}\right)$ coatings. These were obtained by atomic layer deposition (ALD), providing conformal, uniform thickness films on the surface of our nanopillars with strong, covalent bonding to the surface. We observed concurrent opposing blue and red-shifts with the thinnest depositions of $\mathrm{Al}_{2} \mathrm{O}_{3}$, with shift direction dictated by the spectral position of the bare $\mathrm{SPhP}$ mode relative to the ordinary LO phonon of $\mathrm{Al}_{2} \mathrm{O}_{3}$, in case, a blue-shift for modes localized at frequencies higher than the LO phonon and a red-shift for modes localized below it. The concurrent shifts persisted for thicker deposited films and are interpreted as a consequence of the coupling to the characteristic transverse mode observed near the LO frequency of thin dielectric films, named

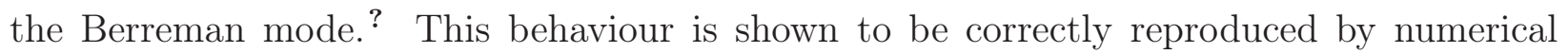
simulations employing the measured permittivity of the deposited $\mathrm{Al}_{2} \mathrm{O}_{3}$ films. On the other hand, the deposition of $\mathrm{ZrO}_{2}$ lead to blue-shifts of longitudinal and transverse SPhP resonances spectrally localized around $900 \mathrm{~cm}^{-1}$. The anomalous shifts persisted for layers up to $\approx 1.5 \mathrm{~nm}$ in thickness, reversing to the pure red-shift expected for a dielectric screening of resonances when the nanostructures were covered with thicker layers. This result cannot be explained by finite element simulations utilising the measured dielectric functions of 
the $\mathrm{ZrO}_{2}$ films excluding diffractive effects, ? implying the influence of some microscopic process. To quantitatively explain the observed shifts we introduce a localized surface state modelled as a Lorentz oscillator-permittivity which may represent a covalent bonding of the deposited film with the $\mathrm{SiC}$ surface, whose response dominates over the bulk optical response of the layer for sufficiently thin films. We demonstrate that such a theoretical model does indeed predict such reversible shifts when the pole of the surface state oscillator is spectrally located between the SPhP resonances of the SiC nanostructure. Such an approach results in interactions that can be interpreted as a consequence of strong coupling. With SPhPs being a strong candidate for resonant SEIRA-based sensing approaches, our results imply that ultra-sensitive methods can be realised based on the strong surface sensitivity of the SPhP resonances with even small local changes to the ambient environment, with the magnitude of the anomalous spectral blue-shifts we report providing a key feature for distinguishing the thickness and potentially the chemical nature of the surface bond associated with the coating layer.

\section{Results}

To determine the sensitivity of SPhPs to changes in the surface chemistry and local ambient, nanopillar arrays were fabricated from semi-insulating $4 \mathrm{H}-\mathrm{SiC}$ substrates, using electronbeam lithography and reactive ion etching with details provided in the literature. ? The Reststrahlen band of 4H-SiC lies between the transverse (TO) and longitudinal optic (LO) phonons. Due to the crystalline anistropy of $4 \mathrm{H}-\mathrm{SiC}$, there are actually two spectrally overlapping Reststrahlen bands due to the in- and out-of-plane phonons that occur between 797-969 and 784-964 $\mathrm{cm}^{-1}$, respectively. ${ }^{?}$ In this region the real component of the dielectric function, plotted in Fig. ??a, is negative and nanopillars of the kind studied here support localized sub-diffraction resonances. These narrow linewidth optical modes, separable 

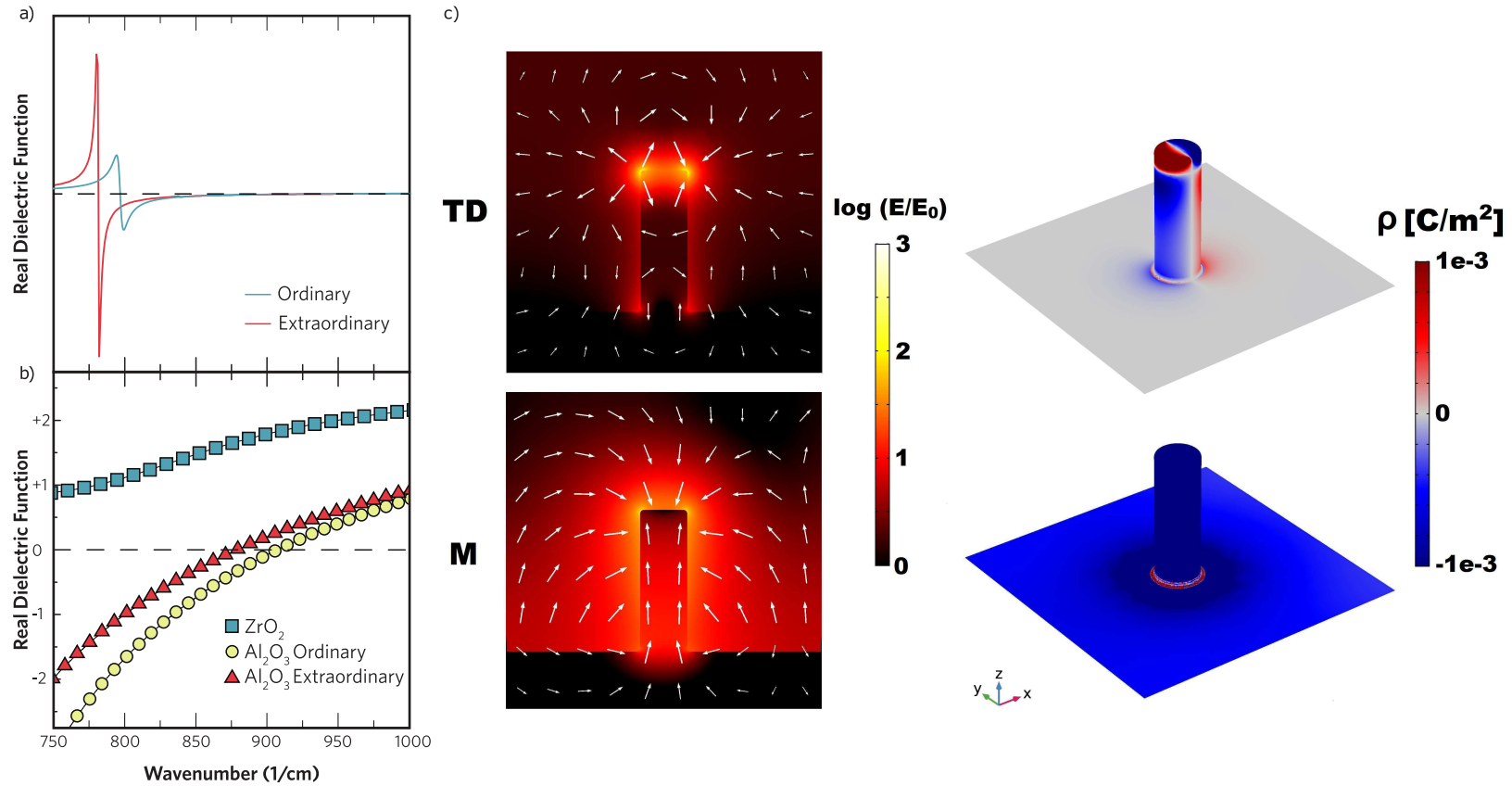

Figure 1: a. The real component of the ordinary (blue) and extraordinary (red) dielectric function of $4 \mathrm{H}-\mathrm{SiC}$ measured by ellipsometry. b. The real component of the dielectric function of $\mathrm{ZrO}_{2}$ (blue squares) and the ordinary (yellow circles) and extraordinary (red triangles) dielectric functions of $\mathrm{Al}_{2} \mathrm{O}_{3}$. c. (left) Cross-section of a nanopillar of diameter $300 \mathrm{~nm}$, height $900 \mathrm{~nm}$ and period $2000 \mathrm{~nm}$ showing the near field (color scale) and electric field polarization (white arrows) of transverse dipolar (TD) and monopolar (M) modes. (right) The respective surface charge density. 
into transverse dipole resonances with electric field orientated perpendicular and monopolar modes with electric field orientated parallel to the cylinder axis, demonstrate huge field enhancements at the resonator surface (Fig ??c). ${ }^{?}$ In order to probe the modes surface sensitivity, we employed Fourier transform infrared (FTIR) reflectance specroscopy of the $\mathrm{SiC}$ nanopillar arrays following iterative ALD of either $\mathrm{Al}_{2} \mathrm{O}_{3}$ or $\mathrm{ZrO}_{2}$. This allowed for the spectral shift to be quantified as a function of the ALD coating thickness, while also providing overall trends. In addition, the choice of two distinct ALD materials offered the demonstration of the generality of the results.

Crystalline $\mathrm{Al}_{2} \mathrm{O}_{3}$ has six Reststrahlen bands in the mid-IR spectral range, each defined by a Coulomb-split pair of TO-LO phonons, with zeros in the permittivity at the LO frequencies. ? The two highest energy bands are delimited by in- and out-of-plane LO modes at $\approx 906.6 \mathrm{~cm}^{-1}$ and $\approx 881.1 \mathrm{~cm}^{-1}$, respectively, both definining positive values for the corresponding ordinary and extraordinary permittivities for frequencies higher than the LO modes themselves. Measurements of the permittivity of thin $\mathrm{Al}_{2} \mathrm{O}_{3}$ films grown on flat $4 \mathrm{H}-$ SiC substrates (Fig ??b) show good agreement with the reported values, even though ALD provides amorphous layers and the optical constants may not be invariant for ultrathin films $(<5 \mathrm{~nm}) .^{?}$ Therefore, $\mathrm{Al}_{2} \mathrm{O}_{3}$ is not expected to provide the dielectric screening leading to

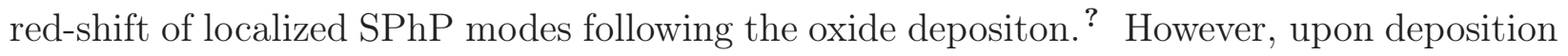
of a $0.35 \mathrm{~nm}$ thick layer of $\mathrm{Al}_{2} \mathrm{O}_{3}$, a red-shift of the monopolar (M) and a blue-shift of the transverse-dipolar (TD) mode were observed (bare resonances at $895 \mathrm{~cm}^{-1}$ and $938 \mathrm{~cm}^{-1}$, respectively, dashed lines in Fig. ??b), as shown for a $300 \mathrm{~nm}$ diameter and $700 \mathrm{~nm}$ period array, a behaviour which persisted for thicker films. Notably, the ordinary LO phonon of the $\mathrm{Al}_{2} \mathrm{O}_{3}$ film lies between both resonances, shown as a vertical dotted line in Fig. ??b. Numerically calculated reflectance of a pillar array of same diameter and pitch incorporating the ordinary permittivity of $\mathrm{Al}_{2} \mathrm{O}_{3}$ as the ALD coating show the same trend in monotonic shifts of the bare resonator modes (Fig. ??a), with direction dependence on whether the bare mode lies above or below the ordinary $\mathrm{Al}_{2} \mathrm{O}_{3} \mathrm{LO}$ phonon frequency at $\approx 906.6 \mathrm{~cm}^{-1}$ (vertical 

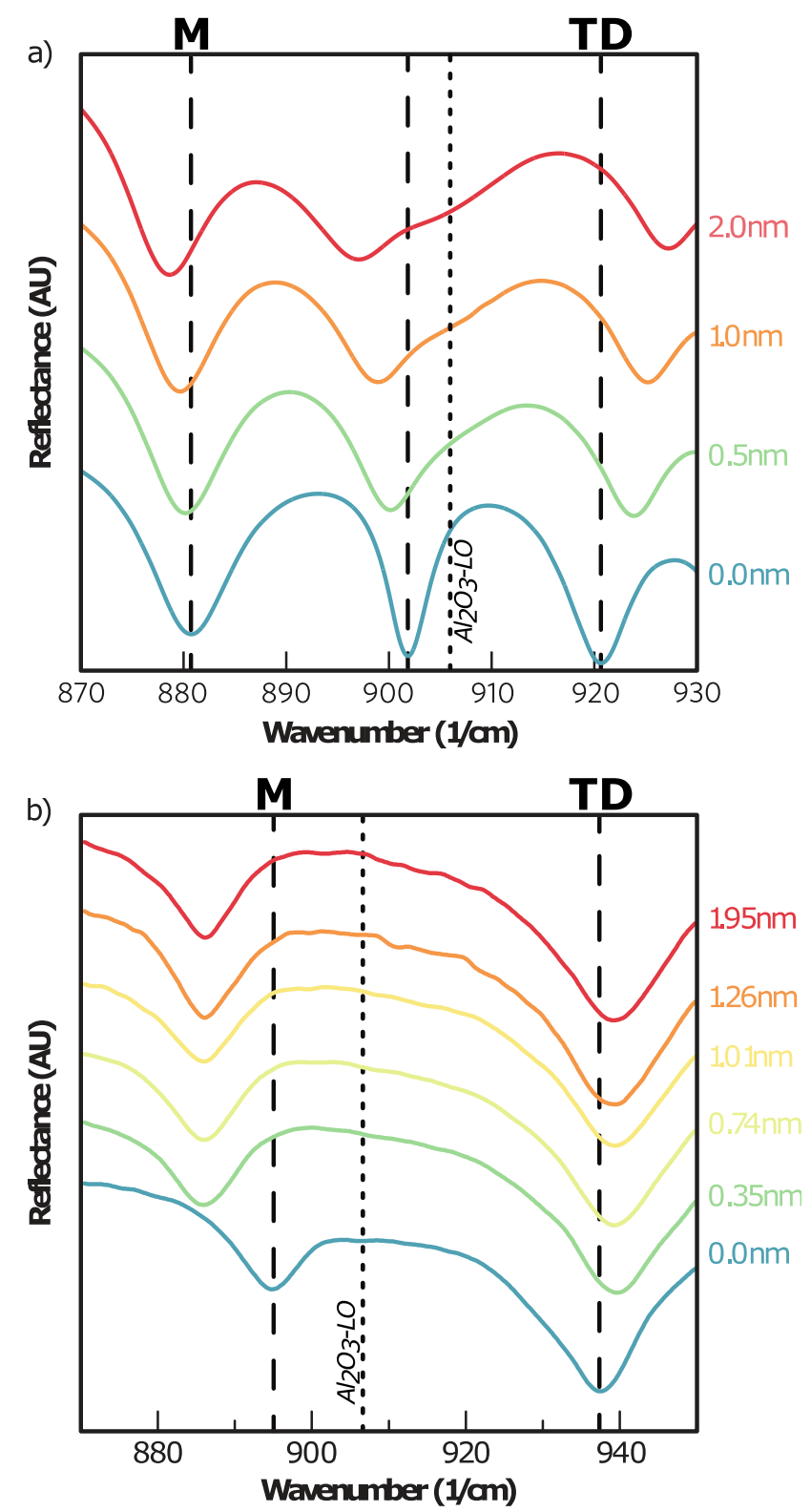

Figure 2: a) Numerical reflectance of an array of $4 \mathrm{H}-\mathrm{SiC}$ nanopillars of diameter $300 \mathrm{~nm}$, height $900 \mathrm{~nm}$ and period $700 \mathrm{~nm}$. b) Experimental reflectance of an array of $4 \mathrm{H}-\mathrm{SiC}$ nanopillars of diameter $300 \mathrm{~nm}$, height $900 \mathrm{~nm}$ and period $700 \mathrm{~nm}$. All are shown for varying $\mathrm{Al}_{2} \mathrm{O}_{3}$ overlay thicknesses. 
dotted line). ${ }^{?}$ The additional resonance at $\approx 902 \mathrm{~cm}^{-1}$ observed in simulations corresponds to a longitudinal mode of the pillar, which, lying below the ordinary $\mathrm{Al}_{2} \mathrm{O}_{3} \mathrm{LO}$ phonon, also red-shifts upon the film deposition. Experimentally this mode is merged with the TD mode, being spectrally resolved for larger pitches (see Supplementary Fig. S2).

It is known that crystalline and amorphous thin films of dielectric materials exhibit sharp resonances in transmission/reflection of p-polarized light near their TO and LO phonons, the former due to TO modes being IR-active and the latter due to a phenomenon named the Berreman effect. ? The corresponding Berreman mode is a dispersive, leaky (wavevectors within the vacuum light cone) transverse mode in nature, excited by the normal compontent of the electric field relative to the film at frequencies slighly above the LO phonon of the material, where its positive dielectric function vanishes. ${ }^{?}$ Although being able to propagate to the far-field, the near-zero permittivity of the film allows this mode to provide huge subdiffractional confinement of the incident radiation. Thus, as a consequence of the extreme confinement of the resonator $\mathrm{SPhP}$ near the $4 \mathrm{H}-\mathrm{SiC}$ surface, it is reasonable to interpret that the observed mode shifts occur due to their hybridisation with the Berreman mode excited at frequencies slightly higher than the ordinary LO phonon of $\mathrm{Al}_{2} \mathrm{O}_{3}$.

Further evidence of the coupling to the Berreman mode is shown in Fig. ??a, which illustrates the effect of a $0.35 \mathrm{~nm} \mathrm{Al} \mathrm{Al}_{2} \mathrm{O}_{3}$ coating on the monopolar mode, which lies at lower frequencies than the Berreman mode, for a variety of nanopillar diameters. The bare reflectance is illustrated by the solid lines, and the coated by the dashed lines. In all cases deposition of an $\mathrm{Al}_{2} \mathrm{O}_{3}$ film results in red shifted modal frequencies of the monopole. The larger shifts for smaller diameter pillars are a consequence of the reduced mode volumes and larger surface confinement provided by those. ${ }^{\text {? }}$ Also noteworthy in this data is the strong coupling achieved between the resonator monopolar mode and the zone-folded LO phonon of the $4 \mathrm{H}-\mathrm{SiC}$ substrate, near $837 \mathrm{~cm}^{-1}\left(\mathrm{P}_{1}\right.$ mode - vertical dashed line in Fig. ??a), an example of the hybridisation observed between localized SPhP and modes of the patterned substrate, ${ }^{?}$ which is also influenced by the shifts induced by the film deposition. The results 
for modes lying above and between the extraordinary and ordinary LO phonons of the film shown in Fig. ??b are less clear as the resonator arrays support many spectrally overlapping modes in this region. Nonetheless it is clear that modes lying below the ordinary LO phonon frequency at $\approx 906.6 \mathrm{~cm}^{-1}$ (vertical dotted line in Fig. ??b) red-shift, while those lying above blue-shift, inferring that the dominant hybridisation is to the leaky mode near the ordinary LO phonon, again being more prominent for pillars of smaller diameter. Notably, the monopolar mode of the $500 \mathrm{~nm}$ diameter, $700 \mathrm{~nm}$ pitch resonator array, whose bare energy lies slightly above the LO phonon mode, also blue-shifts. This might indicate a shifted epsilon-near-zero condition of the Berreman mode to higher frequencies due to a change in the effective permittivity of the thin film, induced by a chemical mechanism, by a substrate roughness comparable to the layer thickness, or even by a shift of the ordinary LO phonon due to mechanical stresses generated from the thermal expansion coefficient mismatch between $\mathrm{Al}_{2} \mathrm{O}_{3}$ and $4 \mathrm{H}-\mathrm{SiC}$ ? However, due to the amorphous nature of ALD growth, the impact of stresses can be considered minimal. This should be considered in systems where epitaxial growth is included, for instance in thin films grown via molecular beam epitaxy or chemical vapour deposition. It is important to note that the transverse, photonic modes of the resonator cannot directly hybridise with the longitudinal mode of the film in the absence of hydrodynamic effects which are not included in our numerical simulations.? These further corroborate the hybridisation between the SPhP and the leaky Berreman mode of the film, resulting from surface charges induced in the film by the out-of-plane component of the electric field provided by the localized modes. ?

Contrasting with $\mathrm{Al}_{2} \mathrm{O}_{3}$, measurements of $\mathrm{ZrO}_{2}$ films $(>3 \mathrm{~nm}$ ) deposited on a $4 \mathrm{H}-\mathrm{SiC}$ substrate have shown that $\mathrm{ZrO}_{2}$ has a relatively flat and uniformly $\geq 1$ permittivity across the Reststrahlen band of 4H-SiC (illustrated by the blue squares in Fig. ??b), which should provide a pure dielectric screening and shift to lower energies of all SPhP resonances upon thin film coating. However, contrary to prior work, following the deposition of ultrathin $\mathrm{ZrO}_{2}$ films (5 cycles, $0.37 \mathrm{~nm}$ ) an anomalous blue-shift is observed for $\mathrm{SPhP}$ resonances of both 

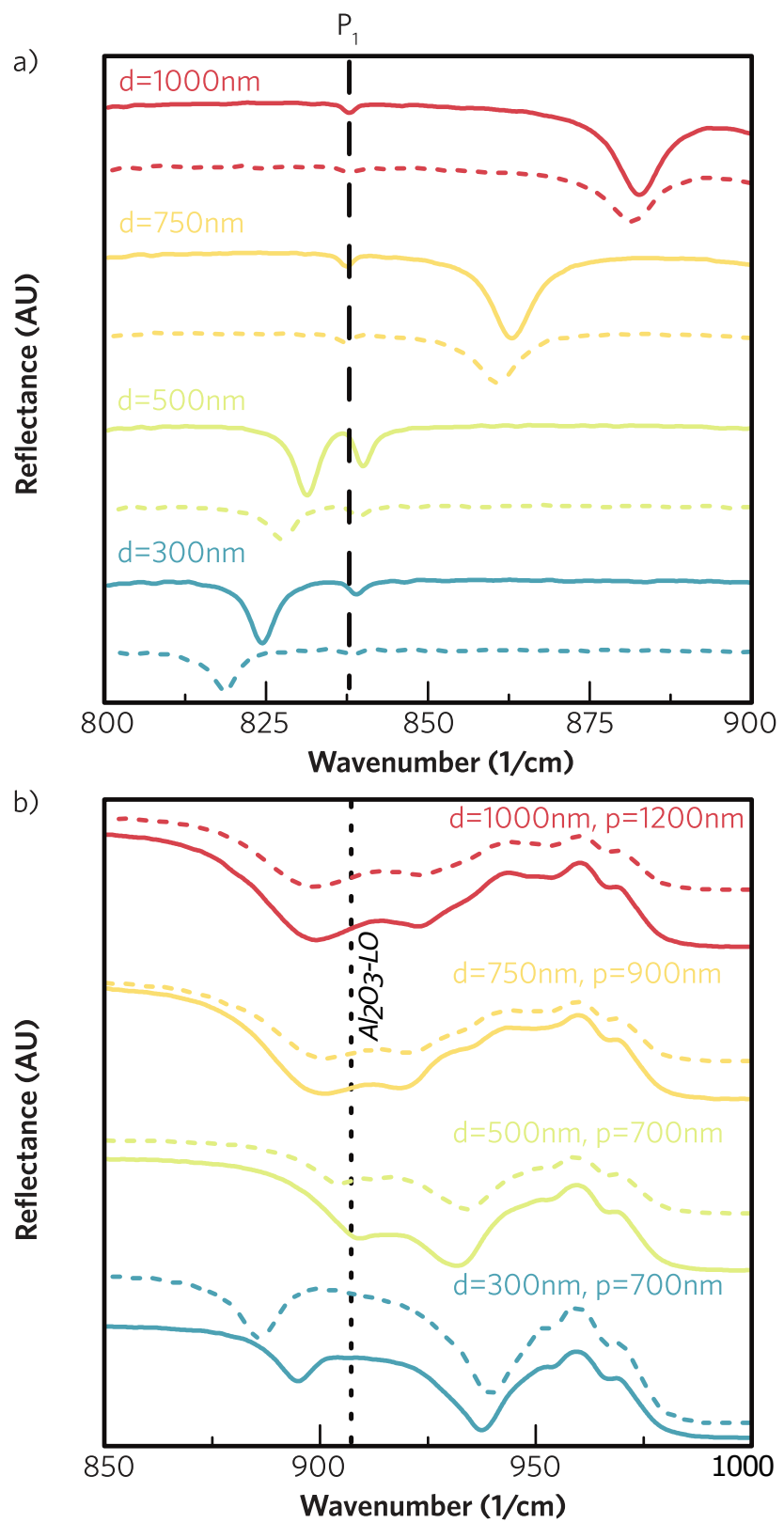

Figure 3: a) Experimental reflectance from an array of 4H-SiC nanopillars of indicated diameter, height $900 \mathrm{~nm}$ and period $2000 \mathrm{~nm}$. b) Experimental reflectance from an array of $4 \mathrm{H}-\mathrm{SiC}$ nanopillars of the diameter and period indicated and height $900 \mathrm{~nm}$. All are shown for the bare array (solid lines) and for a $0.35 \mathrm{~nm} \mathrm{Al}_{2} \mathrm{O}_{3}$ coating (dashed). Ordinary LO phonon of $\mathrm{Al}_{2} \mathrm{O}_{3}$ shown as a vertical dotted line 

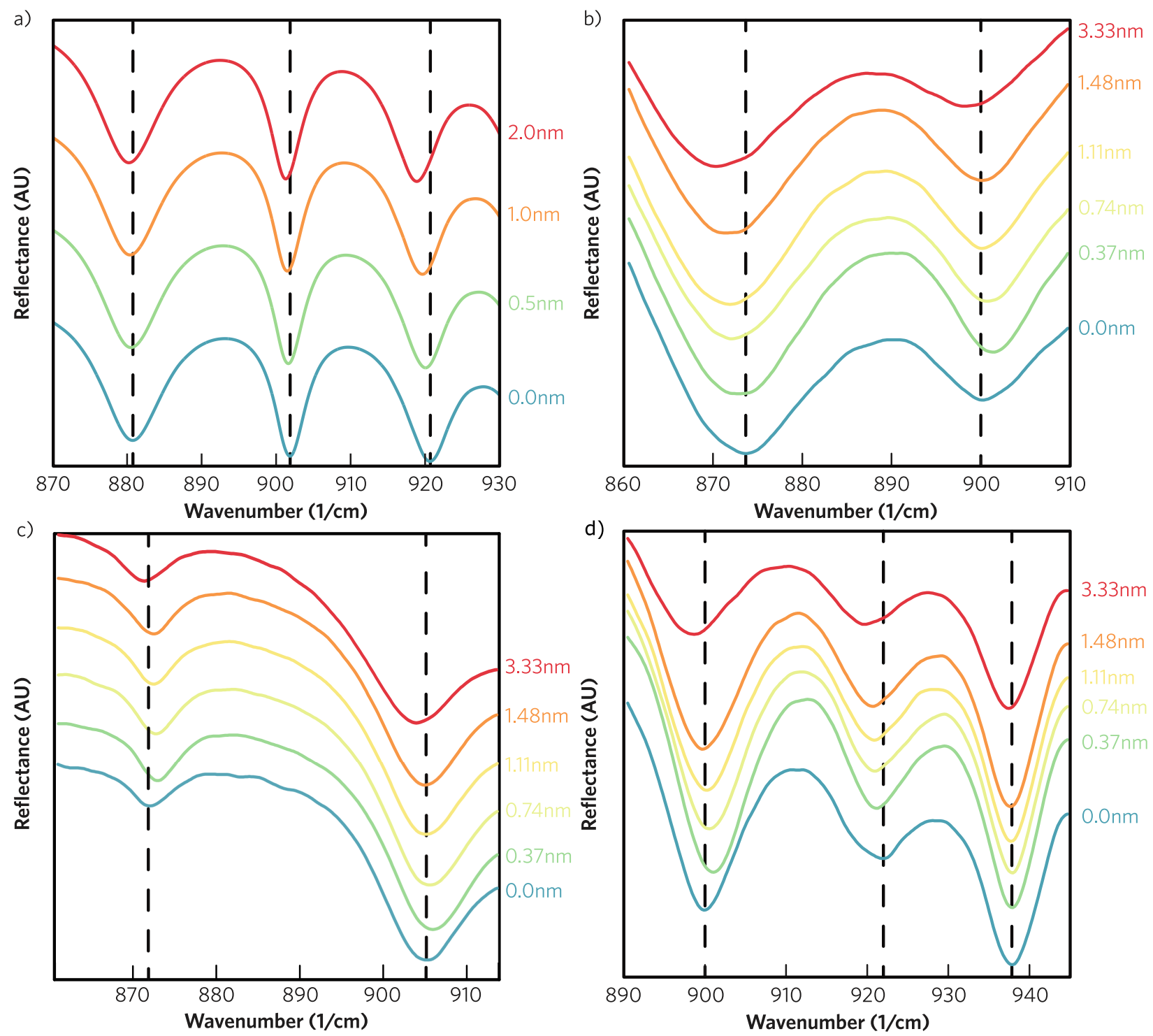

Figure 4: a) Numerical reflectance of an array of 4H-SiC nanopillars of diameter 300nm, height $900 \mathrm{~nm}$ and period $700 \mathrm{~nm}$. b) Experimental reflectance of an array of 4H-SiC nanopillars of diameter $300 \mathrm{~nm}$, height $900 \mathrm{~nm}$ and period $400 \mathrm{~nm}$. c) Experimental reflectance of an array of 4H-SiC nanopillars of diameter $300 \mathrm{~nm}$, height $900 \mathrm{~nm}$ and period $700 \mathrm{~nm}$. d) Experimental reflectance of an array of 4H-SiC nanopillars of diameter $500 \mathrm{~nm}$, height 900 $\mathrm{nm}$ and period $1500 \mathrm{~nm}$. All are shown for varying $\mathrm{ZrO}_{2}$ overlay thicknesses. 
longitudinal and transverse-dipolar character near $900 \mathrm{~cm}^{-1}$, as illustrated for different $\mathrm{SiC}$ nanopillar arrays in Fig. ??b-d. With subsequent depositions, the resonances initially continue this anomalous shift, however, after 20 deposition cycles $(\approx 1.5 \mathrm{~nm}$ thick film) this shift is reversed and the anticipated red-shift is induced and continues with further film growth. For an array of nanopillars of diameter $300 \mathrm{~nm}$ and period $400 \mathrm{~nm}$ (Fig. ??b), a monotonic red-shift is observed for the resonance at $873 \mathrm{~cm}^{-1}$ while the anomalous reversible blue-shift is observed for the one at $900 \mathrm{~cm}^{-1}$. The small periodicity for this array does not allow an unique assignment of a monopolar or transverse-dipolar character to these resonances, due to mode overlapping and hybridization at small gaps. ${ }^{\text {Fig. }}$ ??c shows the anomalous behaviour for both the monopolar $\left(872 \mathrm{~cm}^{-1}\right)$ and the transverse-dipolar $\left(905 \mathrm{~cm}^{-1}\right)$ resonances of a $300 \mathrm{~nm}$ diameter and $700 \mathrm{~nm}$ period array. Similar results are observed for an array of nanopillars of diameter $500 \mathrm{~nm}$ and array period $1500 \mathrm{~nm}$, as shown in Fig. ??d. Here monotonic red-shifts are observed for the modes with bare energies of $922,938 \mathrm{~cm}^{-1}$, correspoding to higher-order transverse modes induced by corners and edges of the pillars, as well as a reversible blue-shift seen for the lowest order transverse-dipolar mode with bare energy of $900 \mathrm{~cm}^{-1}$. This anomalous behaviour was not reproduced by employing the measured permittivity of $\mathrm{ZrO}_{2}$ films deposited on flat $4 \mathrm{H}-\mathrm{SiC}$ substrates. This is illustrated in Fig. ??a, which shows the numerically calculated reflectance of the same pillar array considered previously, whose three dominant modes red shift monotonically as thicker coats of $\mathrm{ZrO}_{2}$ are applied.

The shift of resonances with the thinnest $\mathrm{ZrO}_{2}$ deposition layer was further investigated by exploring the tunability of localized SPhP with the array periodicity. The resultant spectral shifts for $300 \mathrm{~nm}$ diameter nanopillar arrays are plotted as a function of the original peak position of the uncoated structure in Fig. ??a and for $500 \mathrm{~nm}$ diameter nanopillar arrays in Fig ??b. From this it is clear that regardless of the array geometry, anomalous shifts are spectrally localized around $900 \mathrm{~cm}^{-1}$.

To eliminate the effect of altered diffractive coupling as a result of the presence of the $\mathrm{ZrO}_{2}$ 

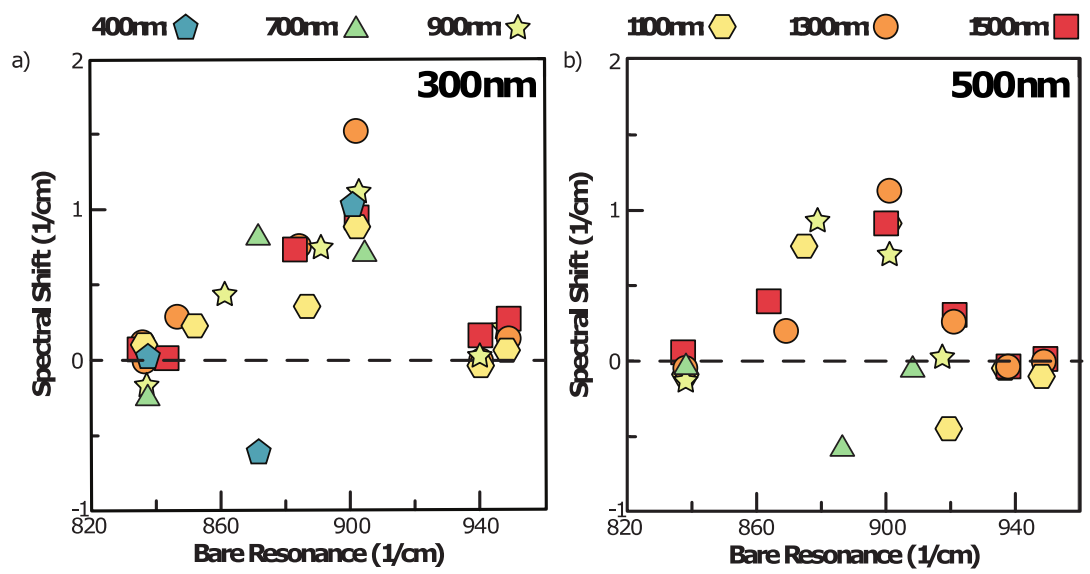

Figure 5: Relative shift as a function of original peak position for a $0.37 \mathrm{~nm}$ thick $\mathrm{ZrO}_{2}$ layer deposited on arrays of nanopillars of a) diameter $300 \mathrm{~nm}$ and height $900 \mathrm{~nm}$ b) diameter 500 $\mathrm{nm}$ and height $900 \mathrm{~nm}$ for varying array pitch.
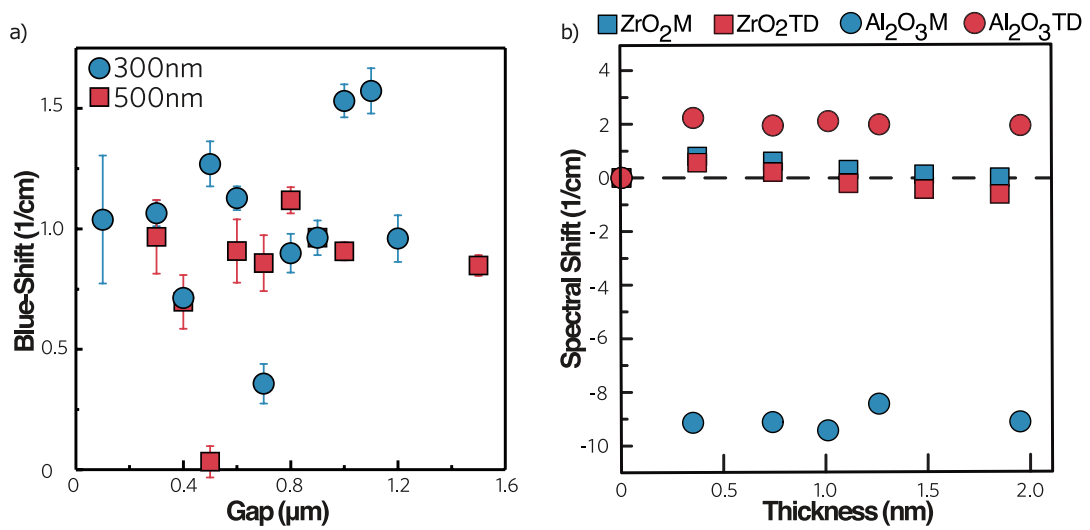

Figure 6: a) Blue shift as a function of gap for nanopillars of diameter $300 \mathrm{~nm}$ (circles) and $500 \mathrm{~nm}$ (squares). b) Relative shifts of the monopole (M) and transverse-dipole (TD) of the $300 \mathrm{~nm}$ diameter and $700 \mathrm{~nm}$ period pillar array as a function of the thickness of $\mathrm{Al}_{2} \mathrm{O}_{3}$ and $\mathrm{ZrO}_{2}$ layers. 
layer the blue shift of the transverse dipolar mode for a $0.37 \mathrm{~nm}$ thick coating was calculated for each lattice period, and is shown as a function of gap size (equal to the array period minus the pillar diameter) in Fig. ??a. No clear dependence of the blue-shift upon the interpillar distance was observed, implying that the anomalous blue-shift is not mediated by near-field interaction between neighbouring pillars and originates from some other mechanism. The magnitude and rate of shifts of the monopolar (M) and transverse-dipolar (TD) modes of the 300nm diameter and 700nm period array as a function of $\mathrm{Al}_{2} \mathrm{O}_{3}$ and $\mathrm{ZrO}_{2}$ thickness is shown in Fig. ??b, corresponding to the spectra in Fig. ??b and Fig. ??c, respectively. A steady red-shift of both modes from the initial $\approx 1 \mathrm{~cm}^{-1}$ blue-shift can be observed for thicker $\mathrm{ZrO}_{2}$ deposited layers, while opposite shifts of larger magnitude, especially for the monopole, are obtained from the initial $\mathrm{Al}_{2} \mathrm{O}_{3}$ deposition. Although the high reflectivity of $\mathrm{Al}_{2} \mathrm{O}_{3}$ within its Reststrahlen bands might prevent the accurate determination of peaks, the large initial shifts and the relative invariance with thicker layers might indicate that mechanisms other than the dielectric function of $\mathrm{Al}_{2} \mathrm{O}_{3}$ and the coupling to the Berreman mode may influence the shift behaviour. Nevertheless, the opposite trends relative to the ordinary LO phonon as obtained with calculations is maintained for thicker films. To determine the role of the Zr precursor (Zirconium (IV) tert-butoxide) utilised in the ALD process, additional spectra were recorded after five deposition cycles, but without incorporating the iterative exposure to the oxygen precursor, resulting in monotonic red-shifts. This resulted only in small, red-shift in the resonance frequencies (Supplementary Fig. S1a,b). The potential of non-uniform pillar coverage or modified film morphology as the primary cause for the anomalous spectral shifts was also eliminitated, as control samples with zirconia layers of each of the same thicknesses were also grown in a single ALD deposition and provided quantitatively similar results as the those from the iterative depositions described above (Supplementary Fig. S1c). This is further evidenced through atomic force microscopy (AFM) analysis of $\mathrm{ZrO}_{2}$ films deposited on a $4 \mathrm{H}-$ $\mathrm{SiC}$ substrate, where conformal coating occurs even over substrate imperfections leading to a smooth surface (RMS roughness $<0.5 \mathrm{~nm}$ ) for thicker ALD layers (Supplementary Fig. S3). 


\section{Discussion}

The experimental data for $\mathrm{Al}_{2} \mathrm{O}_{3}$ qualitatively follows the results of our simple numerical calculations, utilising the measured bulk-like dielectric function to describe the oxide film, even though its optical constants are expected to change for sub-nanometric thick layers. The observed hybridisation between the resonator modes and the leaky mode at frequencies of vanishing dielectric function near the ordinary LO phonons of the film presents a method to predictively tune the spectral landscape of a SPhP resonator system. In addition it demonstrates the potential of the strong near-field confinement of SPhP for sensing applications as large spectral shifts are observed for oxide film thicknesses of just $0.35 \mathrm{~nm}$ for which the sensitivity of macroscopic sensing methods such as ellipsometry is insufficient, especially for absorbing ultrathin films where a strong correlation between the film thickness and permittivity prevent their unique determination.?

The blue shifts observed for $\mathrm{ZrO}_{2}$ however remain anomalous, not being explained by our simple model employing the measured permittivity of the $4 \mathrm{H}$-SiC-deposited $\mathrm{ZrO}_{2}$ and suggesting the influence of external effects. Studies on ultrathin $\mathrm{SiO}_{2}$ films have shown the formation of a transition layer of different stoichiometry $\left(\mathrm{SiO}_{x}, \mathrm{x} \neq 2\right)$, shape and mechanical stress between the substrate and the deposited oxide, leading to an overall increase in the dielectric function in the visible range of the film when reducing its thickness, ${ }^{?}$ a phenomenon which could lead to substantial deviations of the permittivity of sub-nanometric layers of $\mathrm{ZrO}_{2}$ in the mid-IR. The presence of a native $\mathrm{SiO}_{2}$ layer and its influence on the ALD growth of the $\mathrm{Al}_{2} \mathrm{O}_{3}$ and $\mathrm{ZrO}_{2}$ films was evaluated experimentally through X-ray photoelectron spectroscopy (XPS) and also through numerical simulations (Supplementary Fig. S4 and Fig. S5). Although reported in the literature, ${ }^{?}$ XPS analysis show no indication of a native layer on the surface of the $4 \mathrm{H}-\mathrm{SiC}$. In addition, no mixing between the substrate with the ALD 
films is observed. Calculated effects of an eventual $1 \mathrm{~nm} \mathrm{SiO}_{2}$ layer show a pure red-shift of modes due to dielectric screening and also no alteration in the induced behaviour by a second $0.5 \mathrm{~nm}$ cover layer of either $\mathrm{ZrO}_{2}$ or $\mathrm{Al}_{2} \mathrm{O}_{3}$ (Supplementary Fig. S5). Coordination defects in incomplete deposited layers could lead to a rearrangement of atoms in the structure of the thin film in the form of surface reconstructions, leading to properties different than displayed by the bulk material. This could influence the propagation of phonons in the underlying crystal and to shifts of the SPhP modes. However, the deposition of amorphous films through ALD and the random distribution of coordination defects in the layer are not expected to generate a well-defined reorganization of the film and to unidirectional $\mathrm{SPhP}$ mode shifting, an effect that could be more prominent if epitaxial growth techniques for crystalline layers were employed. ' In addition, surface reconstructions in $4 \mathrm{H}-\mathrm{SiC}$ would occur for temperatures above $900^{\circ} \mathrm{C}$, much higher than the $200^{\circ} \mathrm{C}$ used here for ALD. Nevertheless, in order to explain experimental results we introduce a process analogous to that observed for $\mathrm{Al}_{2} \mathrm{O}_{3}$, namely hybridisation with a spectrally interstitial resonance. The source of this resonance is assumed to be microscopic and localized to the interface between the $4 \mathrm{H}-\mathrm{SiC}$ and the initial deposition of $\mathrm{ZrO}_{2}$. Physically such a resonance could stem from either a vibrational bond between the $4 \mathrm{H}-\mathrm{SiC}$ and either the $\mathrm{ZrO}_{2}$ or residual precursor constituents, a vanishing effective permittivity induced by surface roughness of the substrate leading to a leaky-mode coupling, or from an interface dipole. ${ }^{\text {T }}$ The elucidation of the nature of such surface state may be properly addressed by ab initio techniques and is outside the scope of this work. Regardless of its microscopic origin, the interstitial resonance can be effectively modeled by an additional homogeneous layer, characterised by isotropic dielectric function

$$
\epsilon(\omega)=\epsilon_{\infty}\left[1+\frac{f \omega_{0}^{2}}{\omega_{0}^{2}-\omega^{2}+i \gamma \omega}\right]
$$

where $\epsilon_{\infty}$ is the high-frequency dielectric constant, $\omega_{0}$ is the transverse frequency of the proposed resonance and $f$ the resonance oscillator strength. 
To model the effect numerically, we consider a thin boundary layer of thickness $0.5 \mathrm{~nm}$

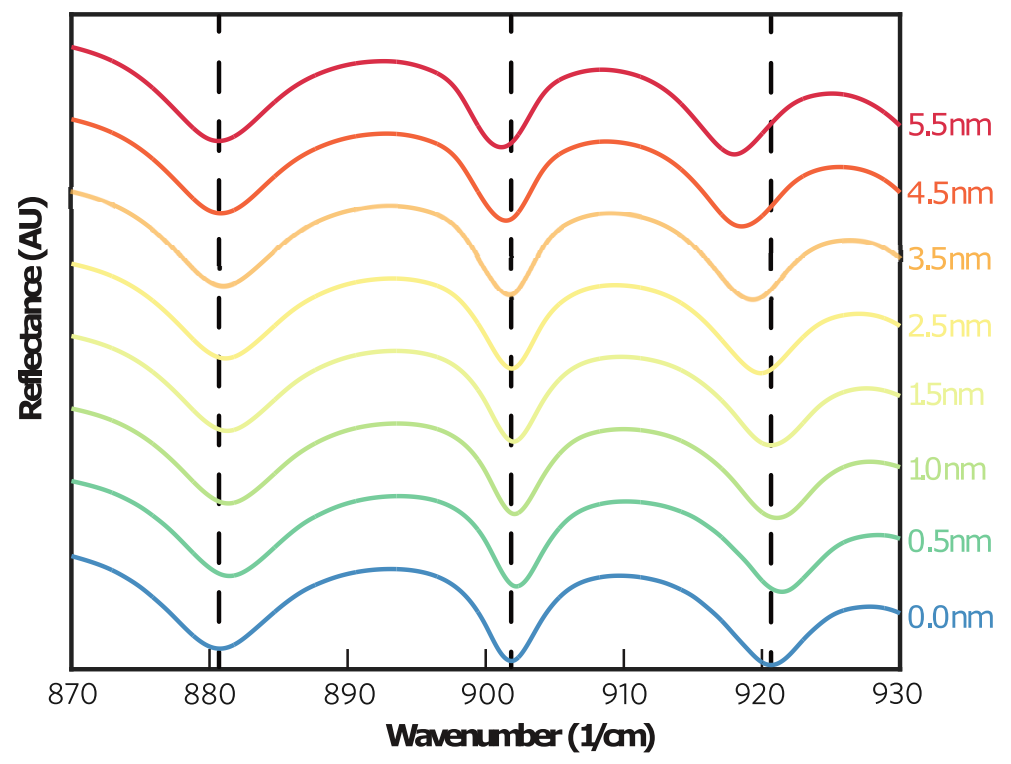

Figure 7: Numerical reflectance of a 300nm diameter, 900nm height and 700nm nanopillar array of $4 \mathrm{H}-\mathrm{SiC}$ coated with a $0.5 \mathrm{~nm}$ layer of resonant dielectric parameterised by $\epsilon_{\infty}=2$, $f=1.2, \omega_{0}=580 \mathrm{~cm}^{-1}, \gamma=2 \mathrm{~cm}^{-1}$ and a layer of $\mathrm{ZrO}_{2}$. The total coating thickness is indicated.

with resonant dielectric function of form eq. ?? parameterised as $\epsilon_{\infty}=2, f=1.2, \omega_{0}=580$ $\mathrm{cm}^{-1}, \gamma=2 \mathrm{~cm}^{-1}$, whose longitudinal (LO) resonance therefore lies at $860 \mathrm{~cm}^{-1}$, placed between the $4 \mathrm{H}-\mathrm{SiC}$ resonator and the deposited $\mathrm{ZrO}_{2}$ film, which are both described by their bulk dielectric functions. Numerical reflectance, for the same pillar array considered previously, is shown in Fig. ?? for SPhP resonances above the interstitial layer LO frequency, reproducing the blue shifts of all modes after the first 5 deposition cycles as a result of coupling to the interstitial resonance, followed by weaker monotonic red shifts as a result of the bulk $\mathrm{ZrO}_{2}$ film. This denotes a transition in the overall optical response from the one dominated by anomalous shifts that can be interpreted as a strong-coupling between the SPhP resonances with the LO resonance of the interstitial layer to the one where the dielectric screening provided by thick $\mathrm{ZrO}_{2}$ overwhelms the interstitial layer effect. This behaviour is consistent with reported for the decreased interaction between interface phonons with thicker layers in heterostructures. A localized surface state stemming from a vibrational bond or an interface dipole might also be present in the case of $\mathrm{Al}_{2} \mathrm{O}_{3}$ deposition, but its effect may be 


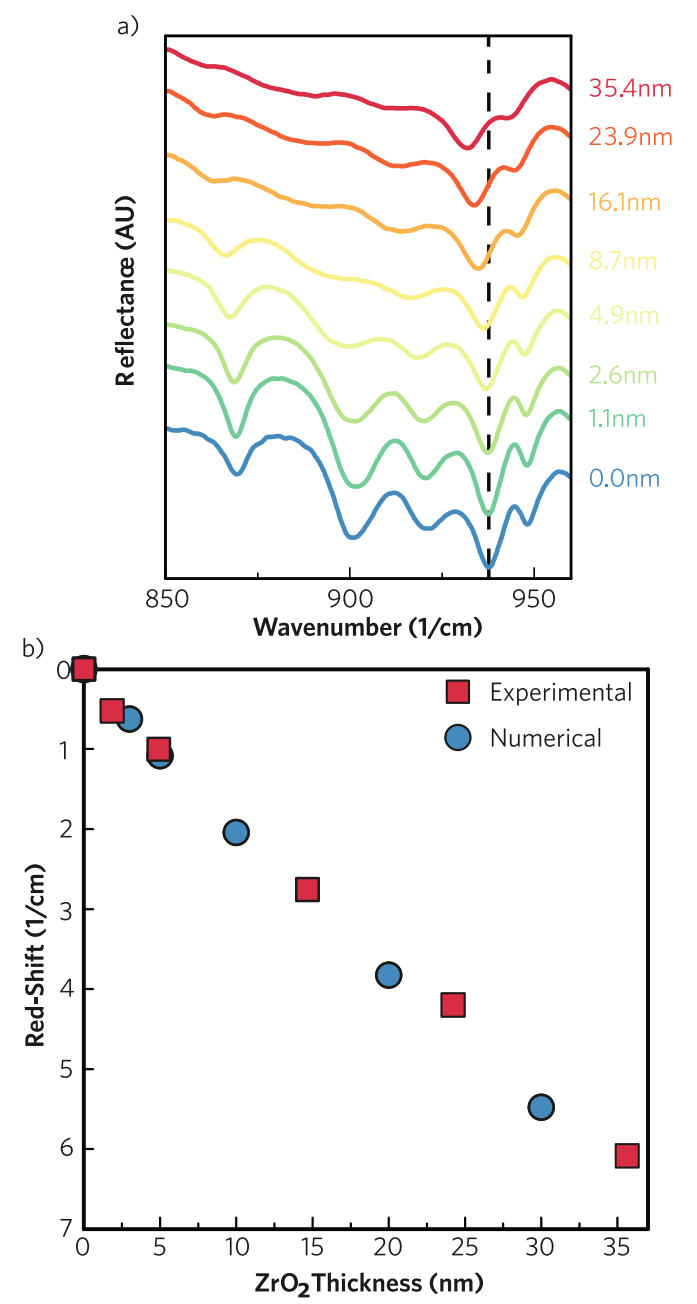

Figure 8: Experimental reflectance from an array of $500 \mathrm{~nm}$ diameter nanopillars of 1300 $\mathrm{nm}$ period for the labelled thickness of $\mathrm{ZrO}_{2}$ encapsulation. Dashed line indicate the bare frequency of the mode, whose shift is plotted as a function of $\mathrm{ZrO}_{2}$ thickness as squares in b). Circles indicate predictions from finite element simulations. 
overshadowed by the interaction with the Berreman mode of the thin film.

Finally we investigated the ability of full 3D finite element method (FEM) calculations to predict spectral shifts as a function of the deposited film thickness. Raw spectra for selected peaks of nanopillars of diameter $500 \mathrm{~nm}$ and period $1300 \mathrm{~nm}$ is shown in Fig. ??a. For thicker films the monotonic red shifts predicted by numerical calculations are observed, allowing for comparison with experimental results. The squares in Fig. ??b track the shifts of the higher-order pillar mode whose bare frequency is indicated by the dashed line in Fig. ??a as a function of film thickness. Circles indicate the corresponding numerical prediction. Both show a monotonic red-shift, and good agreement is reached considering the nominal fabrication values used for the geometry in numerical calculations. This given array period was chosen following a better correspondence observed by FEM predictions for larger pitches, where the pillar modes are spectrally separated.

\section{Conclusions}

In this paper we investigated the interaction of SPhPs supported by $4 \mathrm{H}-\mathrm{SiC}$ nanopillar arrays with ultrathin oxide films deposited by $\mathrm{ALD}$. The results for $\mathrm{Al}_{2} \mathrm{O}_{3}$ showed hybridisation between the tightly confined resonator modes and the leaky Berreman mode at frequencies slightly above the ordinary LO phonon of the oxide layer, in accordance with numerical modelling results. For $\mathrm{ZrO}_{2}$ films the anomalous spectral shifts observed for films of up to $\approx 1.5 \mathrm{~nm}$ in thickness could not be explained by macroscopic electrodynamic calculations utilising the measured dielectric function. The anomalous shifts are explained as a result

of hybridisation of the SPhPs with a phenomenological phononic or electronic interface mode, however, a deeper understanding of this mechanism necessitates the use of ab initio methods such as density functional theory. The strong spectral shifts observed, even for atomically thin films, demonstrate the potential of tightly confined SPhP modes for sensing applications. Moreover the predictable nature of the shifts for thicker films, which are in 
accordance with numerical expectations for thicknesses greater than $3 \mathrm{~nm}$, may present an alternative for real-time thin film deposition monitoring. This work offers new prospects to the field of nanophotonics, exploring new light-matter interaction regimes of vibrational and optical modes and offering a new sensing platform in the mid-IR using low-loss plasmonic-like materials.

\section{Methods}

\section{Nanofabrication of SiC Nanopillar Arrays}

Silicon Carbide pillar arrays were etched into semi-insulating $4 \mathrm{H}-\mathrm{SiC}$ substrates. $\mathrm{Al} / \mathrm{Cr}$ hard masks defining the geometry of the pillar array were deposited on the substrate through electron-beam lithography, lift-off and evaporation techniques. The pillar height was defined by the exposure time of a subsequent reactive ion etch, where the masked substrate was exposed for 38 minutes at $150 \mathrm{~W}$ utilising $\mathrm{SF}_{6}$ and $\mathrm{Ar}$ in equal partial pressures, followed by a chemical wet etch. In order to remove any residual fluorine a commercial PlasmaSolv treatment was performed.

\section{Atomic layer deposition}

Conformal and highly uniform oxide film growth was performed in an Ultratech Savannah 200 reactor with a chamber temperature of $200^{\circ} \mathrm{C}$ and a base pressure of 0.1 Torr prior to precursor injection. Sequential pulsing and purging of individual precursors was optimized to allow a homogeneous coverage of the hig aspect-ration nanostructures. Zirconium (IV) tert-butoxide (ZTB) and trimethylaluminum (TMA) were utilised as precursors for zirconia and alumina films respectively, while deionized water acted as the oxygen source in both cases. Cation and oxygen precursors of oxide films were injected alternately in the ALD chamber, defining one deposition cycle, and providing the self-limiting reactions leading to film growth. Linear growth rates were obtained for both oxides, with averaged deposition 
rates of $0.074 \mathrm{~nm} /$ cycle for $\mathrm{ZrO}_{2}$ and $0.108 \mathrm{~nm} /$ cycle for $\mathrm{Al}_{2} \mathrm{O}_{3}$. To monitor the ALD process, a p-type $\mathrm{Si}$ witness sample was placed along the $\mathrm{SiC}$ in the reaction chamber for subsequent film thickness evaluation by ellipsometry.

\section{Ellipsometry}

Ellipsometry was employed to characterise the optical constants and thicknesses of deposited films. A J.A. Woollam alpha-SE spectrographic ellipsometer was utilised to collect the spectra across three diferent angles. Refractive index and film thickness were obtained by fitting the data with a Cauchy model. Film growth was obtained from measurements on the aforementioned Si witness sample. To reduce changes in optical properties due to lattice mismatch, oxide film spectra used for calculation were obtained from layers deposited over flat $4 \mathrm{H}-\mathrm{SiC}$ substrates.

\section{Fourier Transform Infrared Spectroscopy}

Infrared measurements were performed in reflectance mode using a Thermo Scientific, Nicolet FTIR Continuum Microscope. A $15 \times$, 0.58 NA reverse Cassegrain objective provided illumination at angles from $10-35^{\circ}$ off-normal, with weighted average of $25^{\circ}$. Spectra were taken as an average of 32 scans with $0.5 \mathrm{~cm}^{-1}$ resolution acquired from a $50 \mu \mathrm{m}^{2}$ area.

\section{Numerical calculations}

Frequency-domain, full-wave solutions of Maxwell's equations were obtained using the RF module of the finite-element solver Comsol Multiphysics. Plane-wave illumination was set at $25^{\circ}$ (corresponding to the weighted-average illumination angle of the objective used in experiments) for a p-polarized incident field (transverse-magnetic mode). Bloch-periodic boundary conditions were applied to represent the periodicity of the arrays. Calculations were performed using the permittivities of $4 \mathrm{H}-\mathrm{SiC}, \mathrm{ZrO}_{2}$ and $\mathrm{Al}_{2} \mathrm{O}_{3}$ as determined by ellip- 
sometry.

\section{References}

(1) Gerard, J. M.; Sermage, B.; Gayral, B.; Legrand, B.; Costard, E.; Thierry-Mieg, V. Enhanced spontaneous emission by quantum boxes in a monolithic optical microcavity. Phys. Rev. Lett. 1998, 81, 1110-1113.

(2) Houdré, R.; Stanley, R. P.; Oesterle, U.; Ilegems, M.; Weisbuch, C. Room-temperature cavity polaritons in a semiconductor microcavity. Phys. Rev. B 1994, 49, 16761.

(3) Maier, S. A. Plasmonics: Fundamentals and Applications. Springer: New York, 2007.

(4) Kneipp, K.; Wang, Y.; Kneipp, H.; Perelman, L. T.; Itzkan, I.; Dasari, R.; Feld, M. S. Single molecule detection using surface-enhanced Raman scattering (SERS). Phys. Rev. Lett. 1997, 78 , 1667-1670.

(5) Khurgin, J. How to deal with the loss in plasmonics and metamaterials. Nat. Nanotechnol. 2015, 10, 2-6.

(6) Khurgin, J.; Sun, G. Comparative analysis of spasers, vertical-cavity surface-emitting lasers and surface-plasmon-emitting diodes. Nat. Photonics 2014, 8, 468-473.

(7) Oulton, R. F.; Sorger, V.; Genov, D. A.; Pile, D. F. P.; Zhang, X. A hybrid plasmonic waveguide for subwavelength confinement and long-range propagation. Nat. Photonics 2008, 2, 496-500.

(8) Oulton, R. F.; Plasmonics: Loss and gain. Nat. Photonics 2012, 6, 219-221.

(9) Caldwell, J. D.; Lindsay, L.; Giannini, V.; Vurgaftman, I.; Reinecke, T. L.; Maier, S. A.; Glembocki, O. J. Low-loss, infrared and terahertz nanophotonics using surface phonon polaritons. Nanophotonics 2015, 4, 44-68. 
(10) Mutschke, H.; Andersen, A. C.; Clement, D.; Henning, T.; Peiter, G. Infrared properties of $\mathrm{SiC}$ particles. Astron. Astrophys. 1999, 345, 187-202.

(11) Caldwell, J.D.; Kretinin, A.V.; Chen, Y.; Giannini, V.; Fogler, M.M.; Francescato, Y.; Ellis, C.T.; Tischler, J.G.; Woods, C.R.; Giles, A.J.; Hong, M.; Watanabe, K.; Taniguchi, T.; Maier, S.A.; Novoselov, K.S. Sub-diffractional volume-confined polaritons in the natural hyperbolic material hexagonal boron nitride. Nat. Commun. 2014, 5, 5221.

(12) Dai, S.; Fei, Z.; Ma, Q.; Rodin, A. S.; Wagner, M.; McLeod, A. S.; Liu, M. K.; Gannett, W.; Regan, W.; Watanabe, K.; Taniguchi, T.; Thiemens, M.; Dominguez, G.; Neto, A. H. Castro; Zettl, A.; Keilmann, F.; Jarillo-Herrero, P.; Fogler, M. M.; Basov, D. N. Tunable Phonon Polaritons in Atomically Thin van der Waals Crystals of Boron Nitride. Science 2014, 343, 1125-1129.

(13) Urzhumov, Y. A.; Korobkin, D.; Neuner, B.; Zorman, C.; Shvets, G. Optical properties of sub-wavelength hole arrays in SiC membranes. J. Opt. A: Pure App. Opt. 2007, 9, S322-S333.

(14) Taubner, T.; Korobkin, D.; Urzhumov, Y.; Shvets, G.; Hillenbrand, R. Near-field microscopy through a SiC superlens. Science 2006, 313, 1595-1595.

(15) Dai, S.; Ma, Q.; Andersen, T.; Mcleod, A.S.; Fei, Z.; Liu, M.K.; Wagner, M.; Watanabe,K.; Taniguchi, T.; Thiemens, M.; Keilmann, F.; Jarillo-Herrero, P.; Fogler M.M.; Basov, D.N. Subdiffractional focusing and guiding of polaritonic rays in a natural hyperbolic material. Nat. Commun. 2015, 6, 6963.

(16) Li, P.N.; Lewin, M.; Kretinin, A.V.; Caldwell, J.D.; Novoselov, K.S.; Taniguchi, T.; Watanabe, K.; Gaussmann, F.; Taubner, T. Hyperbolic phonon-polaritons in boron nitride for near-field optical imaging and focusing. Nat. Commun. 2015, 6, 7507.

(17) Anderson, M. S. Enhanced infrared absorption with dielectric nanoparticles. Appl. Phys. Lett. 2003, 83, 2964-2966. 
(18) Gubbin, C. R.; De Liberato, S. Theory of Nonlinear Polaritonics: $\chi^{(2)}$ Scattering on a $\beta$-SiC Surface. ACS Phot. 2017, 4, 1381-1388.

(19) Gubbin, C. R.; De Liberato, S. Theory of Four-Wave-Mixing in Phonon Polaritons. ACS Phot. 2018, 5, 284-288.

(20) Caldwell, J. D.; Glembocki, O. J.; Francescato, Y.; Sharac, N.; Giannini, V.; Bezares, F. J.; Long, J. P.; Owrutsky, J. C.; Vurgaftman, I.; Tischler, J. G.; Wheeler, V. D.; Bassim, N. D.; Shirey, L. M.; Kasica, R.; Maier, S. A. Low-Loss, Extreme Subdiffraction Photon Confinement via Silicon Carbide Localized Surface Phonon Polariton Resonators. Nano Lett. 2013, 13, 3690-3697.

(21) Chen, Y. G.; Francescato, Y.; Caldwell, J. D.; Giannini, V.; Mass, T. W. W.; Glembocki, O. J.; Bezares, F. J.; Taubner, T.; Kasica, R.; Hong, M. H.; Maier, S. A. Spectral Tuning of Localized Surface Phonon Polariton Resonators for Low-Loss Mid-IR Applications. ACS Photonics 2014, 1, 718-724.

(22) Gubbin, C. R.; Martini, F.; Politi, A.; Maier, S. A.; De Liberato, S. Strong and Coherent Coupling between Localized and Propagating Phonon Polaritons. Phys. Rev. Lett. 2016, $116,246402$.

(23) Gubbin, C. R.; Maier, S. A.; De Liberato, S. Theoretical investigation of phonon polaritons in SiC micropillar resonators. Phys. Rev. B 2017, 95, 035313.

(24) Bohren, C. F.; Huffman, D. R. Absorption and scattering of light by small particles. Wiley-VCH: Weinheim, 2004; pp 331-348.

(25) Harbecke, B.; Heinz, B.; Grosse, P. Optical Properties of Thin Films and the Berreman Effect. Appl. Phys. A 1985, 38, 263-267.

(26) Schubert, M., Tiwald, T. E.; C. M. Herzinger. Infrared dielectric anisotropy and phonon modes of sapphire. Phys. Rev. B 2000, 61, 8187. 
(27) Losurdo, M.; Bergmair, M.; Bruno, G.; Cattelan, D.; Cobet, C.; de Martino, A.; Fleischer, K.; Dohcevic-Mitrovic, Z.; Esser, N.; Galliet, M.; Gajic, R.; Hemzal, D.; Hingerl, K.; Humlicek, J.; Ossikovski, R.; Popovic, Z. V.; Saxl, O. Spectroscopic ellipsometry and polarimetry for materials and systems analysis at the nanometer scale: state-of-the-art, potential, and perspectives. J. Nanopart. Res. 2009, 11, 1521-1554.

(28) Ellis, C. T.; Tischler, J. G.; Glembocki, O. J.; Bezares, F. J.; Giles, A. J.; Kasica, R.; Shirey, L.; Owrutsky, J. C.; Chigrin, D. N.; Caldwell, J. D. Aspect-ratio driven evolution of high-order resonant modes and near-field distributions in localized surface phonon polariton nanostructures. Sci. Rep. 2016, 6, 32959.

(29) De Wolf, I. Micro-Raman spectroscopy to study local mechanical stress in silicon integrated circuits. Semicond. Sci. Technol. 1996, 11, 139-154.

(30) Roca, E.; Trallero-Giner, C.; Cardona, M. Polar optical vibrational modes in quantum dots. Phys. Rev. B 1994, 49, 13704.

(31) Vassant, S.; Hugonin, J.P.; Marquier, F.; Greffet, J.J. Berreman mode and epsilon near zero mode. Opt. Express 2012, 20, 23971-23977.

(32) Campione, S.; Brener, I.; Marquier, F. Theory of epsilon-near-zero modes in ultrathin films. Phys. Rev. B 2015, 91, 121408.

(33) Wang, Y.; Irene, E.A. Consistent refractive index parameters for ultrathin $\mathrm{SiO}_{2}$ films. J. Vac. Sci. Technol. B 2000, 18, 279-282.

(34) Huang, W.; Shao-Hui, C.; Xue-Chao, L.; Biao, S.; Tian-Yu, Z.; Xi, L.; Cheng-Feng, Y.; Yan-Qing, Z.; Jian-Hua, Y.; Er-Wei, S.; Wen-Hua, Z.; Jun-Fa, Z. Direct observation of nanoscale native oxide on $6 \mathrm{H}-\mathrm{SiC}$ surface and its effect on the surface band bending. App. Phys. Exp. 2012, 5, 105802. 
(35) Scopigno, T.; Steurer, W.; Yannopoulous, S.N.; Chrissanthopoulos, A.; Krisch, M.; Ruocco, G.; Wagner, T. Vibrational dynamics and surface structure of amorphous selenium. Nat. Commun. 2011, 2, 195.

(36) Caldwell, J.D.; Vurgaftman, I.; Tischler, J.G.; Glembocki, O.J.; Owrutsky, J.C.; Reinecke, T.L. Atomic-scale photonic hybrids for mid-infrared and terahertz nanophotonics. Nat. Nanotechnol. 2016, 11, 9-15.

(37) Butler, K.T.; Walsh, A. Ultra-thin oxide films for band engineering: design principles and numerical experiments. Thin Solid Films 2014, 559, 64-68.

\section{Conflict-of-interest Disclosure}

Conflict of interest: The authors declare no competing financial interest.

\section{Acknowledgement}

R.B. acknowledges the Capes Foundation for a Science Without Borders fellowship (Bolsista da Capes - Proc. no BEX 13.298/13-5). R.B. and S.A.M. acknowledge support from the LeeLucas Chair in Physics, the Office of Naval Research Global (Grant No. N62909-16-1-2027) and the Leverhulme Trust. S.D.L. is a Royal Society Research Fellow. S.D.L and C.R.G. acknowledge support from EPSRC Grant No. EP/M003183/1. NRL coauthors acknowledge financial support from the Office of Naval Research administered by the Nanoscience Institute at the Naval Research Laboratory in Washington, D.C.

\section{Supporting Information Available}

Reflectance spectra after zirconium precursor deposition in the absence of the oxygen precursor; reflectance spectra of iterative $v s$ single-step growth of the $\mathrm{ZrO}_{2}$ film. Experimental and 
FEM calculations of spectra of 300nm diameter pillar arrays of different pitches. AFM images and XPS analysis of $\mathrm{ZrO}_{2}$ films deposited on flat 4H-SiC substrates. FEM calculations of effects of a $1 \mathrm{~nm}$ native $\mathrm{SiO}_{2}$ layer on bare $\mathrm{SiC}$ modes and on $\mathrm{Al}_{2} \mathrm{O}_{3}$ and $\mathrm{ZrO}_{2}$ deposition. The supporting information is available free of charge via the Internet at http://pubs.acs.org. This material is available free of charge via the Internet at http://pubs.acs.org/. 


\section{Graphical TOC Entry}

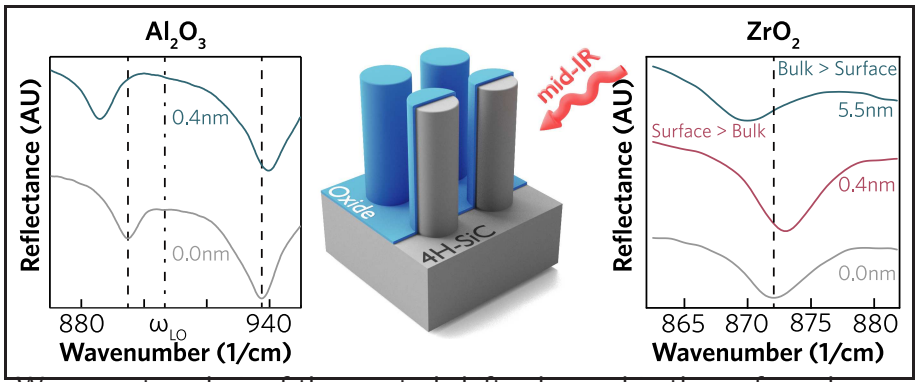

We present analysis of the spectral shifts observed in the surface phonon polariton modes of nano-scale $4 \mathrm{H}-\mathrm{SiC}$ resonators under coating by ultrathin oxide films. The anomalous results indicate the potential of surface phonon polaritons for sensing applications. 